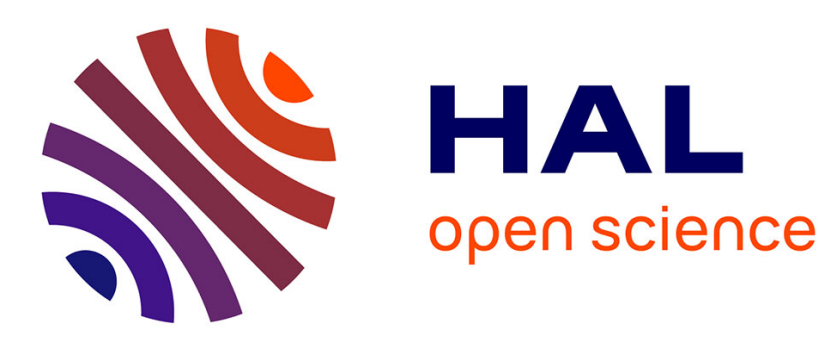

\title{
Dielectric relaxation of Sorbitol revisited
}

Christer Svanberg, Rikard Bergman

\section{To cite this version:}

Christer Svanberg, Rikard Bergman. Dielectric relaxation of Sorbitol revisited. Philosophical Magazine, 2007, 87 (3-5), pp.383-388. 10.1080/14786430600990345 . hal-00513773

\section{HAL Id: hal-00513773 \\ https://hal.science/hal-00513773}

Submitted on 1 Sep 2010

HAL is a multi-disciplinary open access archive for the deposit and dissemination of scientific research documents, whether they are published or not. The documents may come from teaching and research institutions in France or abroad, or from public or private research centers.
L'archive ouverte pluridisciplinaire HAL, est destinée au dépôt et à la diffusion de documents scientifiques de niveau recherche, publiés ou non, émanant des établissements d'enseignement et de recherche français ou étrangers, des laboratoires publics ou privés. 


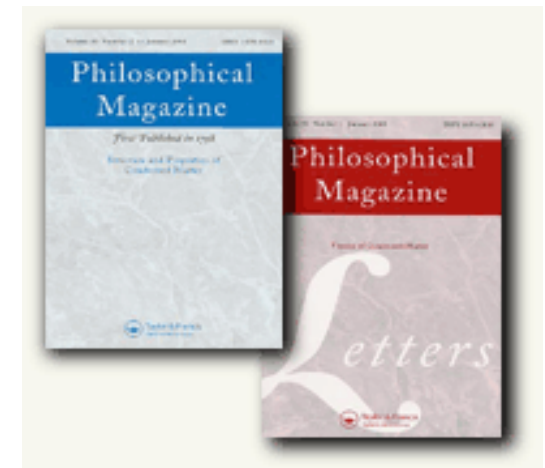

\section{Dielectric relaxation of Sorbitol revisited}

\begin{tabular}{|r|l|}
\hline Journal: & Philosophical Magazine \& Philosophical Magazine Letters \\
\hline Manuscript ID: & TPHM-06-Apr-0089.R2 \\
\hline Dournal Selection: & Philosophical Magazine \\
\hline Author: & 30-Aug-2006 \\
\hline Komplete List of Authors: & $\begin{array}{l}\text { Svanberg, Christer; Chalmers University of Technology, Applied } \\
\text { Physics } \\
\text { Bergman, Rikard; Chalmers University of Technology, Applied } \\
\text { Physics }\end{array}$ \\
\hline Keywords (user supplied): & glass transition, supercooled liquids \\
\hline \multicolumn{2}{|l|}{$\begin{array}{l}\text { Note: The following files were submitted by the author for peer review, but cannot be converted } \\
\text { to PDF. You must view these files (e.g. movies) online. }\end{array}$} \\
\hline svanbergPM06.tex \\
\hline
\end{tabular}

\section{今) ScholaroNE Manuscript Central}


Philosophical Magazine,

Vol. 00, No. 00, DD Month 200x, 1-5

\begin{abstract}
We have performed broad-band dielectric spectroscopy to study the merging of the $\alpha$ - and $\beta$-relaxations in Sorbitol. To investigate this merging in detail the data were analysed both directly in frequency domain and after transforming the data to time domain. At the liquid-glass transition temperature, $T_{g}$, the standard analysis give a clear kink in the temperature dependence of the relaxation time of the $\beta$-relaxation. However, using a more sophisticated analysis we show that the data can be equally well described by extrapolating the low temperature Arrhenius behaviour of the $\beta$-relaxation to the merging region of the $\alpha$ - and $\beta$-relaxations. The Sorbitol data is thus compatible with a scenario with no anomalous temperature behaviour in the merging region.
\end{abstract}

\title{
1 Introduction
}

Much of the attention in the field of glass transition physics has lately been focused on the merging of the main $\alpha$-relaxation and the faster $\beta$-relaxation. The $\beta$-process is often supposed to be rather local compared to the $\alpha$-relaxation, and for polymers it has often been identified with some type of side-group motion. However, Johari and Goldstein [1] showed that molecular glass-formers without intra-molecular degrees of freedom also show dielectric $\beta$-responses. This suggests that $\beta$-relaxations are a general feature of glassy dynamics. Below $T_{g}$ the $\beta$-relaxation has a relaxation time that follows an Arrhenius temperature behaviour. The $\alpha$-relaxation appears to diverge at $T_{0}$ slightly below $T_{g}$ and the relaxation time is normally well described by the VFT equation

$$
\tau=\tau_{0} \exp \left(\frac{D T_{0}}{T-T_{0}}\right)
$$

Here $D$ parameterizes the departure from Arrhenius behavior and is a measure of the fragility of the system [2]. The time decay of the $\alpha$-relaxation is usually well described by the Kohlrausch-Williams-Watts (KWW) equation (see Table 1). In contrast to the $\alpha$-process, the dielectric loss due to a $\beta$-process is normally found to be symmetric on a logarithmic frequency axis and can normally be described using the Cole-Cole equation (CC) (see Table 1). To facilitate analysis in both time and frequency domain we use approximate expressions of the above mentioned functions also in the domain they were not originally defined for (see Table 1 and Refs. [3-6]).

Table 1. Formulas for the KWW and Cole-Cole functions and their respective frequency and time approximations, see Refs. [3-6].

\begin{tabular}{|c|r|r|}
\hline Function & Correlation function, $\phi(t)$ & Permittivity (imag. part), $\varepsilon^{\prime \prime}(\omega)$ \\
\hline "KWW" & $\phi_{0} \exp \left[-(t / \tau)^{\beta}\right]$ & $\frac{\varepsilon_{p}^{\prime \prime}}{1-\beta+\frac{\beta}{1+\beta}\left[\beta\left(\omega_{p} / \omega\right)+\left(\omega / \omega_{p}\right)^{\beta}\right]}$ \\
\hline$" C C "$ & $\frac{\phi_{0}}{1+\sqrt{1-\alpha}(t / \tau)^{\alpha}} \exp \left[-\frac{(t / \tau)^{\alpha}}{1+\sqrt{1-\alpha}(t / \tau)^{\alpha}}\right]$ & $\operatorname{Im}\left[\varepsilon_{\infty}+\frac{\varepsilon_{0}-\varepsilon_{\infty}}{\left[1+\left(i \omega \tau_{C C}\right)^{\alpha}\right]}\right]$ \\
\hline
\end{tabular}

\footnotetext{
*Corresponding author. Email: svanberg@fy.chalmers.se
} 
where $\varepsilon_{s}$ and $\varepsilon_{\infty}$ stand for the static and high frequency limiting values of the dielectric loss, respectively. Eq. 2 was derived without invoking any specific mechanisms for the relaxation processes and is thus very general. The starting point is that the $\beta$-relaxation results from a partial reorientation of the dipole vectors. At longer times it is assumed that the $\alpha$-relaxation independently give a complete reorientation of the dipoles. Neglecting cross-correlation terms the relaxation function in Eq. 2 is obtained. The limitation with a simple superposition ansatz,

$$
\phi(t)=a \phi_{\alpha}(t)+(1-a) \phi_{\beta}(t)
$$

is that it ignores that the $\beta$-process occurs in an environment that is in itself relaxing through the $\alpha$ relaxation, which is certainly better described using Eq. 2. Note that well below $T_{g}$, where $\phi_{\alpha}(t)=1$ in our experimental window, Eqs. 2 and 4 is identical.

In earlier studies $[10-12,15]$ it was shown that using Eq. 2 and extrapolated low temperature values for the relaxation times, the merging could be described with the same low temperature Arrhenius behaviour for the $\beta$-relaxation also in the merging region. In this work we choose to study Sorbitol as it has been subject to several studies that seemingly give evidence for a kink in the temperature dependence of the $\beta$ relaxation time. Since both the $\alpha$ - and the $\beta$-peak is easily detectable using dielectric spectroscopy Sorbitol is well suited for investigation of the merging behavior. It can be shown directly, using the parameters given in literature, that the observed change in activation energy can be explained by using Williams ansatz and the extrapolated low-temperature behaviors [15]. Here we transform (Eq. 3) new high resolution broad band dielectric data to time domain for a more straight forward analysis according to Eq. 2. We also compare the results from a standard superposition analysis.

\section{Results and Discussion}

The dielectric measurements were performed on a Novocontrol system covering a broad frequency range $\left(10^{-2}-10^{9} \mathrm{~Hz}\right)$. The Sorbitol (Aldrich) sample was prepared by placing silica spacers $(0.1 \mathrm{~mm})$ between parallel-plate electrodes. Isothermal spectra were measured from $160 \mathrm{~K}$ to $380 \mathrm{~K}$ with an accuracy better 

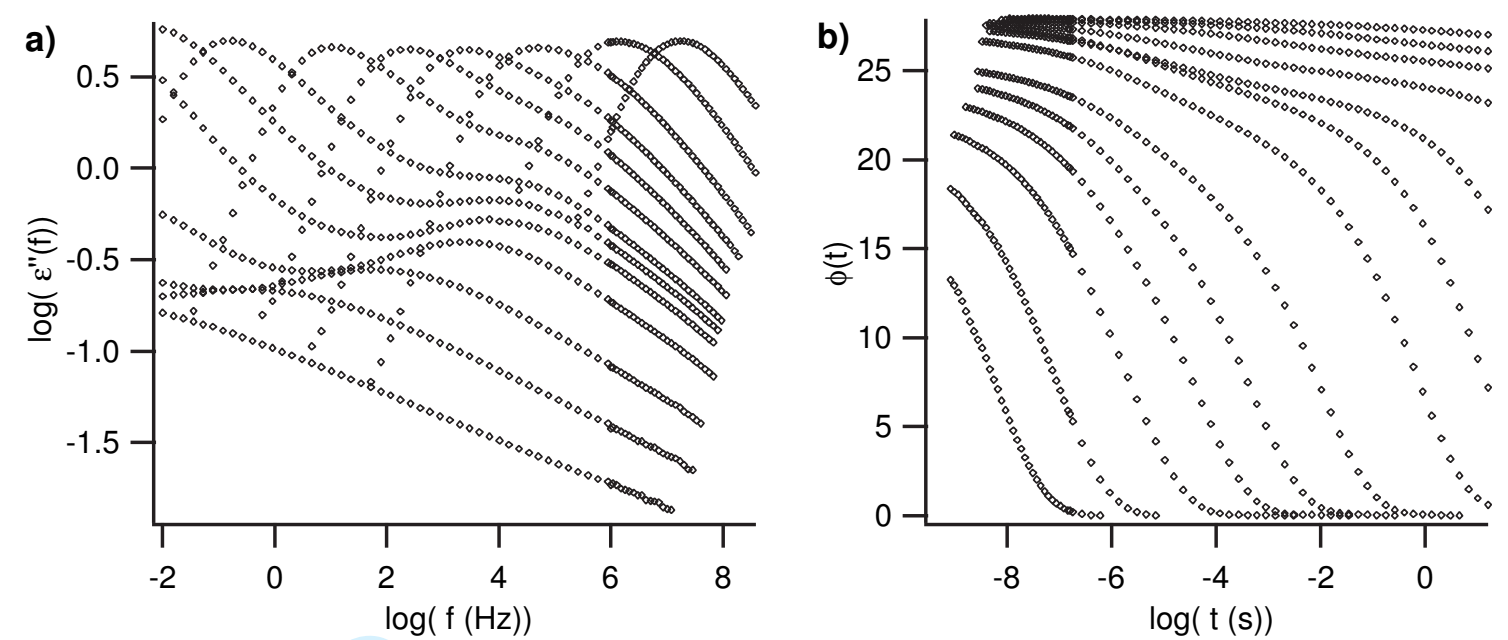

Figure 1. Experimental data at $\mathrm{T}=160,190,220,250,266,269,272,278,284,290,299,314$ and $332 \mathrm{~K}$ from left to right in panel a) and the same but transformed to time domain in panel b).

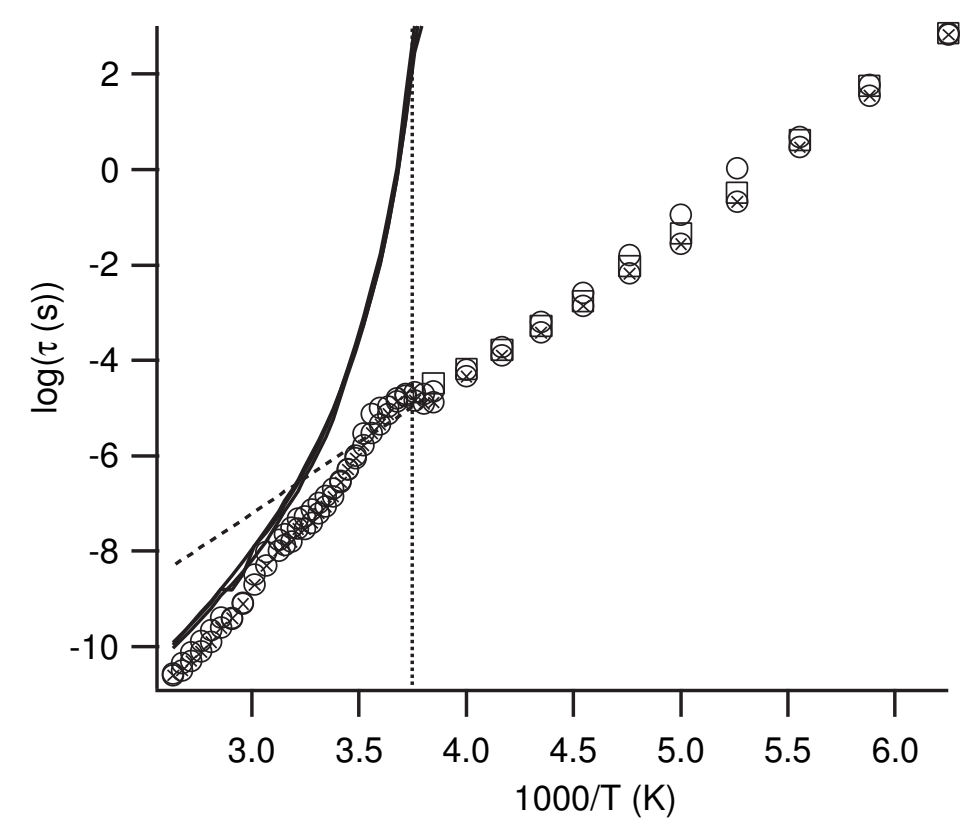

Figure 2. Arrhenius plot of the obtained relaxation times from the different analyzing procedures. The lines represent the different almost indistinguishable $\alpha$-relaxation times. The circles and the crossed circles are representing the $\beta$-relaxation times from the superposition analysis in frequency and time domain, respectively. The squares are the low temperature relaxation time for the data analysed according to Eq. 2 and the dashed line the extrapolation used in the analysis.

than 0.2 K. In Fig. 1a we show some representative dielectric spectra of Sorbitol. The data was first analysed directly in frequency domain using the superposition ansatz (Eq. 4). We here used the CC equation for the $\beta$-relaxation and a frequency-domain version of the KWW equation for the $\alpha$-relaxation (see Table 1). These fits give a good description of the data, but the obtained relaxation times shown in Fig. 2 show the typical temperature dependencies discussed above, i.e. a VFT behaviour for the $\alpha$-relaxation while the $\beta$-relaxation is Arrhenius with an apparent change in activation energy roughly at $T_{g}$.

The ionic conductivity were then subtracted and the data were transformed into time domain according to Eq. 3. The transformed data displayed in Fig. 1b were first fitted with a superposition of a KWW function and a newly derived time-domain version of the CC equation (see Table 1). As seen in Fig. 2 the obtained relaxation times are consistent with the frequency domain fits thus corroborating the transformation method and our fit functions in the frequency and time domains, respectively. We then proceed by fitting 


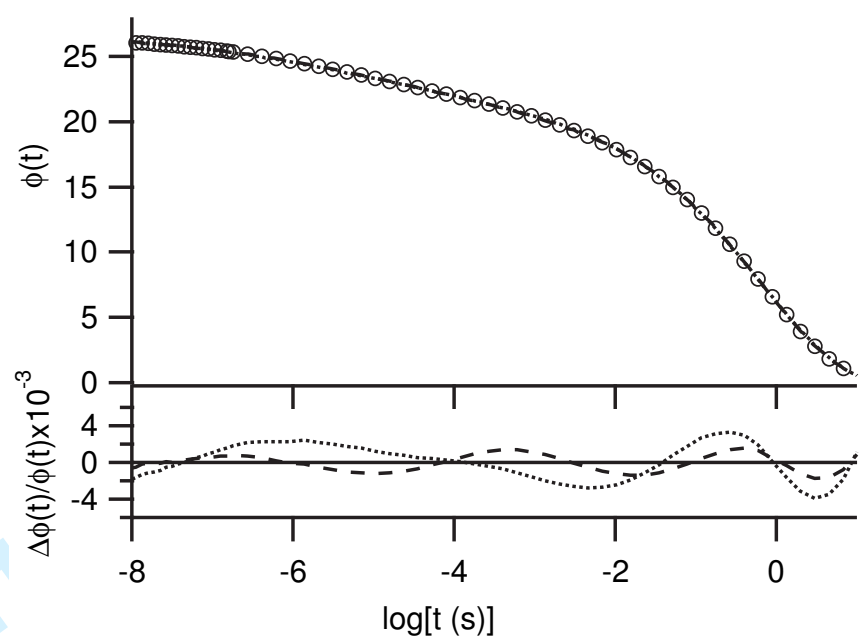

Figure 3. Transformed experimental data at $\mathrm{T}=272 \mathrm{~K}$ and the obtained fits using the superposition (dashed line) and the Williams approach (dotted line). Below the deviations between the data and the fits are shown.

according to Eq. 2 again using a KWW-function for the $\alpha$-relaxation and the time-domain version of the CC-equation for the $\beta$-relaxation. In these fittings the low temperature behaviour of the $\beta$-relaxation (shape and relaxation time) was extrapolated to the merging region. ${ }^{1}$ As seen in Fig. 3 this procedure provides excellent fits to the time domain data in the merging region. The data can thus be described almost equally well with two free parameters less and, most importantly, without the kink in the temperature dependence of the underlying $\beta$-relaxation time.

In Fig. 2 we see that for both analysing approaches we obtain identical temperature-dependence for the relaxation time of the $\alpha$-process. This is well described by the VFT-expression (Eq. 1) with $D=5.2$, $T_{0}=232 \mathrm{~K}$ and $\tau_{0}=4 \times 10^{-14} \mathrm{~s}$, regardless of analysis procedure. For the $\beta$-relaxation below $T_{g}$ the results for the different curve-fit procedures are also very similar. They all follow an Arrhenius behaviour with an activation energy of $62 \mathrm{~kJ} / \mathrm{mol}$. Somewhat above $T_{g}$, however, Fig. 2 shows that the superposition analysis give a change to a higher activation energy. The kink thus happens when the two processes are seemingly rather separated ( $\sim 5$ decades). However, the $\beta$-relaxation is very broad at $T_{g}, \alpha_{C C} \approx 0.3$ gives a FWHM $\approx$ 5 decades [16], and the $\alpha$-relaxation is very stretched towards short times $\left(\beta_{K W W} \approx 0.4\right)$. Thus for Sorbitol the processes start to interfere already slightly above $T_{g}$.

As seen above, the different analysis methods hardly affects the results for neither the $\alpha$-relaxation nor the $\beta$-relaxation below $T_{g}$. However, above $T_{g}$, in the merging region, there is a significant difference of certain importance as it implies different views on the relaxation mechanisms. When taking into account that the dipoles are relaxing in a relaxing environment, the temperature dependence of the low temperature $\beta$-relaxation can be used to describe the dynamics also in the merging region. The analysis according to Williams, Eq. 2, thus gives a physical explanation of the observed kink in the temperature dependence of the $\beta$-relaxation time when analysed with a simple superposition of the $\alpha$ - and $\beta$-relaxation, Eq. 4 . Also note that determining the relaxation time by using the inverse of the peak frequency of the apparent process is practically equivalent to a superposition ansatz. Previously, the merging of the relaxation processes in Sorbitol has been investigated as a function of both pressure and temperature revealing a kink in $\tau_{\beta}(T)$ at, or slightly above, $T_{g}$. [8] Further analysis is required to resolve if also the kink observed at high pressures could be attributed to the interference of the processes.

An additional advantage with the analysis according to Eq. 2 is that, in the not so easily analysed merging region, the number of free parameters can be reduced in the curve-fitting routine. As shown here in the case of Sorbitol we could describe the data equally well with both the shape and the relaxation time of the $\beta$-relaxation locked to the values corresponding to straight forward extrapolation of the easily analysed behaviours found below $T_{g}$. A possible drawback is that the usefulness of time domain representation is

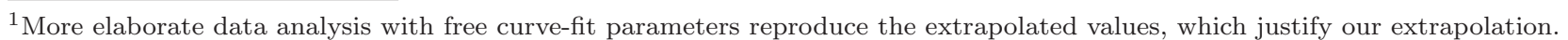


dependent on a rather strong beta-relaxation. In the case of weak $\beta$-relaxations it is probably better to perform the analysis directly in frequency domain as described in Ref. [15].

\section{Conclusions}

We have measured and carefully analysed the dielectric relaxation of Sorbitol with special focus on resolving the seemingly anomalous temperature dependence of the $\beta$-relaxation time. We conclude that the observed kink in the temperature dependence at $T_{g}$ when analyzing with the normal simple superposition is due to the increasing proximity to the $\alpha$-relaxation as temperature is increased. The $\beta$-relaxation then occurs in a relaxing environment and we argue that this is more correctly described by Eq. 2 then by a simple superposition ansatz, Eq. 4. Hence, the full relaxation scenario of Sorbitol is excellently described using Williams Ansatz with the low temperature dependence extrapolated to the merging region.

\section{Acknowledgments}

Financial support from the Swedish Natural Science Research Council is gratefully acknowledged.

\section{References}

[1] G. P. Johari and M. Goldstein. J. Chem. Phys. 53 (1970) 2372.

[2] C. A. Angell. J. Non-Cryst. Solids 131-133 (1991) 13.

[3] G. Williams and D. C. Watts. Trans. Faraday Soc. 66 (1970) 80.

[4] K. S. Cole and R. H. Cole. J. Chem. Phys. 9 (1941) 341.

[5] R. Bergman. J. Appl. Phys. 88 (2000) 1356.

[6] C. Svanberg. J. Appl. Phys. 94 (2003) 4191.

[7] T. Fujima, H. Frusawa and K. Ito. Phys. Rev. E 66 (2002) 031503.

[8] M. Paluch, C. M. Roland, S. Pawlus, J. Ziolo and K. L. Ngai. Phys. Rev. Lett. 91 (2003) 115701.

[9] S. C. Kuebler, D. J. Schaefer, C. Boeffel, U. Pawelzik and H. W. Spiess. Macromolecules 30 (1997) 6597.

[10] F. Alvarez, A. Hoffman, A. Alegria and J. Colmenero. J. Chem. Phys. 105 (1996)(2) 432.

[11] R. Bergman, F. Alvarez, A. Alegria and J. Colmenero. J. Chem. Phys. 109 (1998) 7546.

[12] D. Gomez, A. Alegría, A. Arbe and J. Colmenero. Macromolecules 34 (2001) 503.

[13] G. Williams. Adv. Polymer Sci. 33 (1979) 60.

[14] T. Blochowicz and E. A. Rössler. Phys. Rev. Lett. 92 (2004) 225701.

[15] R. Bergman and C. Svanberg. Phys. Rev. E 72 (2005) 043501.

[16] C. Svanberg and R. Bergman. J. Non-Cryst. Solids 283 (2001) 225. 
Pafyei|రsqfohical Magazinf \& Philosophical Magazine Letters

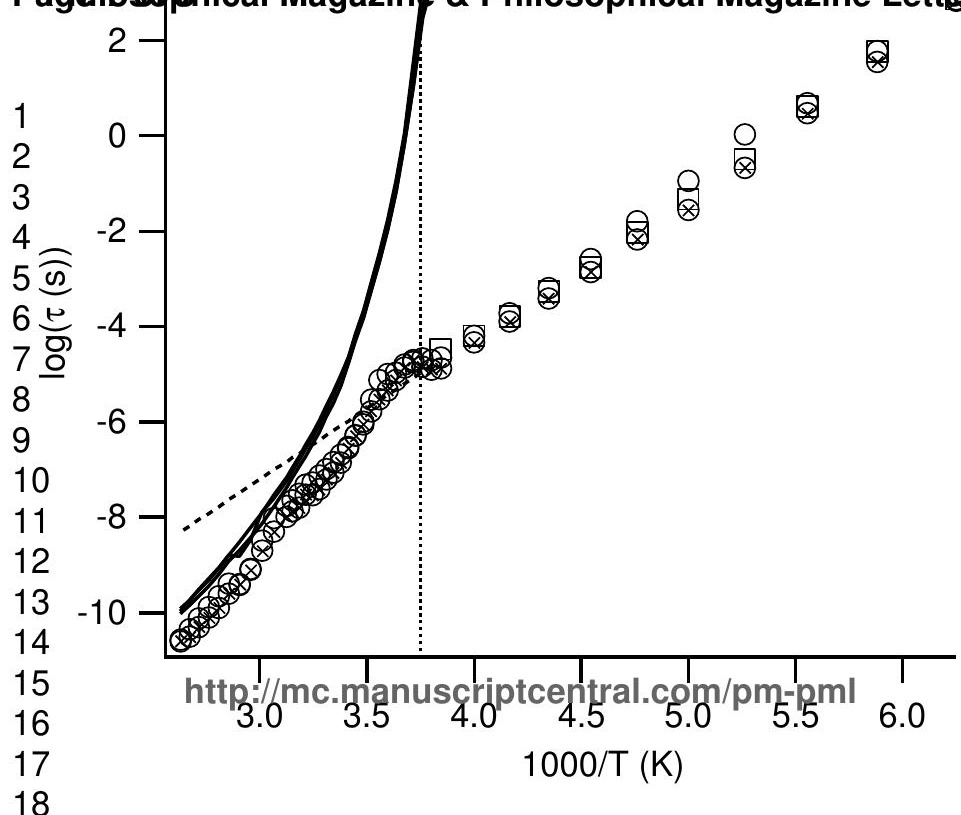


hilosoph hioa Magazine \& Philosophical Maßgagin\& bede

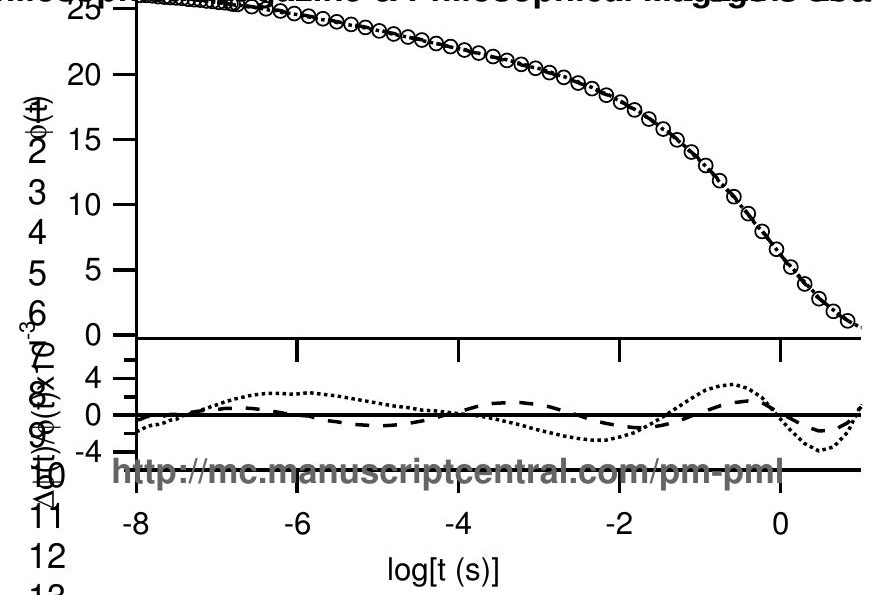

